\title{
Renata Mauperin, la liberación de la feminidad a través del elemento líquido
}

\author{
Aragón-Ronsano, Flavia
}

Universidad de Cádiz. flavia.aragon@uca.es

\section{Resumen}

El inicio de la novela Renata Mauperin de los hermanos Goncourt publicada en 1864 es una escena en la que dos personajes, un hombre y una mujer, están hablando y la joven cuestiona las normas sociales y familiares impuestas a las jóvenes burguesas de su época. Esta escena tiene lugar en el río Sena y sin duda el elemento líquido es el detonante de la liberación de la feminidad artística, emocional y personal encerrada en el personaje de Renata que a su vez está inspirado de mujeres reales que codearon los Goncourt.

Palabras clave: Renata Mauperin; Goncourt; feminidad; liberación; líquido.

\section{Résumé}

L'incipit du roman Renée Mauperin des frères Goncourt publié en 1864 c'est une scène où deux personnages, un homme et une femme, sont en train de parler et la jeune questionne les normes sociales et familiales imposées aux jeunes bourgeoises de son époque. Cette scène a lieu dans la Seine est sans aucun doute l'élément liquide est le détonnant de la libération de la féminité artistique, émotionnelle et personnelle enfermées dans le personnage de Renée qui à la fois est inspiré de femmes réelles que los Goncourt ont fréquentées.

Mots-clés: Renée Mauperin; Goncourt; féminité; libération; liquide.

\begin{abstract}
The beginning of the french novel Renée Mauperin of the Goncourt brothers published in 1864 is a scene where two personages, a man and a woman, are talking and the young woman critics the social and familiars norms imposed to the young girls of bourgeoisie of her time. This scene is happeneing int he Sena river and surely the liquid element is the trigger of the liberation of artistic, emotional and personal feminity inclosed in the personnage of Renée that is inspirated on real women that the Goncourt met.
\end{abstract}

Keywords: Renée Mauperin; Goncourt; féminity; liberation; liquid. 


\title{
Renée Mauperin, 1864
}

En 1859 Edmond y Jules de Goncourt terminan su primera gran novela a cuatro manos, Les hommes de lettres, cuyo protagonista, Charles Demailly, se dedica curiosamente a escribir una novela: La Bourgeoisie. Esta obra ficticia dentro de la verdadera obra, es el modelo exacto de la novela que ambos hermanos están preparando y que hasta 1864 no fue publicada. Renée Mauperin es una de las seis novelas escritas a cuatro manos por Edmond y Jules juntos; ya en 1855 tenían en mente la idea de una novela sobre la burguesía, querían hacer el retrato de toda una clase social a través de caracteres, haciendo corresponder a cada tipo de mujer un tipo de hombre. La idea inicial de los Goncourt era trazar el devenir de tres generaciones de la burguesía, proyecto ambicioso y muy novedoso que se adelantaba al de Emile Zola con los Rougon-Macquart. En este sentido es curioso ver el cambio de título que se va señalando ya en el Journal: de La Bourgeoisie se pasa a La jeune Bourgeoisie, mostrando ya en las notas preparatorias el sacrificio de la primera generación; la primera versión se publicó como Mademoiselle Mauperin y finalmente acabó llamándose Renée Mauperin. Todavía en el prefacio de la reedición de 1875 Edmond duda de la pertinencia de dicho título, pero ya le parece demasiado tarde para modificarlo; queda pues como anécdota si la novela es en realidad el retrato de una clase social o simplemente el retrato de una joven. La crítica y los contemporáneos de los Goncourt acogieron bastante bien Renée Mauperin; Gustave Flaubert, íntimo de Edmond y Jules, en una carta que les envía en febrero del 64, les cuenta su entusiasmo tras la lectura de la novela y se permite incluso hacerles algunas críticas estilísticas:

\author{
Nuit de lundi, 3heures \\ (Paris, 29 février 1864) \\ Mes Bichons, \\ Mademoiselle Bosquet m’écrit pour me demander s'il vous est agréable qu'elle vous fasse un article dans le Journal \\ de Rouen. Elle admire grandement votre dernier livre [Renée Mauperin]. \\ Et moi aussi, car je viens de le lire ou plutôt de le dévorer en entier et d'une seule haleine. Ça m’a charmé. Voilà tout \\ ce que je puis dire maintenant. Ce qui me reste le plus dans la tête, c'est le portrait de l'abbé, celui d'Henri et la mort \\ de Renée. Quel charmant être que cette jeune fille-là ! \\ Ce volume m'a l'air roide, dites-vous ? Je vais maintenant le relire posément. \\ Mais c'est l'exemplaire de Bouilhet que j'ai reçu. Où est le mien? \\ Comme ça s'enchaine ! Quel mouvement ! Et il y a des morceaux chouettes, des portraits classiques. Le dialogue au \\ commencement entre les époux, exquis ; le deuil, superbe, etc. \\ J'ai été irrité plusieurs fois par des imparfaits dans la narration. Sont-ce des fautes typographiques ou bien est-ce \\ intentionnel ? \\ Adieu. Je n'en puis plus ; je vous prends sur ma table de nuit et je vous relis. \\ Tendresses de votre vieux. \\ Oui, sacré nom de Dieu, c'est bien, très bien ! J’ai franchement ri à deux ou trois places et mouillé à quelques autres \\ (comme un bourgeois). Comme vous avez du talent et d'esprit et comme je vous aime ! (Dufief, $1998: 124)^{1}$
}

También hubo opiniones contrarias y duras sobre la novela, dirigidas casi todas al personaje de Renée que la crítica consideró demasiado grosero, escandaloso e inconsecuente ${ }^{2}$. Sin embargo la novela tuvo mucho éxito, tanto en Francia como en el extranjero, y once años más tarde (el joven Jules había fallecido) Renée Mauperin se reeditó ${ }^{3}$ y esta vez Edmond de Goncourt añadió un prefacio ${ }^{4}$ muy revelador en cuanto a la declaración de intenciones de los autores y sobre las motivaciones que les llevaron a cambiar de título: aclara el interés por el análisis psicológico del personaje, el estilo realista y depurado utilizado para la narración, es decir, todo lo que a nivel formal define el texto goncourtiano y

\footnotetext{
${ }^{1}$ A su muerte Flaubert tenía en su biblioteca un ejemplar de la edición Charpentier de 1864, con la dedicatoria: «À Gustave Flaubert/ ses amis/ Edmond et Jules de Goncourt» (Dufief, 1998: 125).

${ }^{2}$ En este sentido Pierre-Jean Dufief afirma: «la critique reprochera souvent au personnage de Renée, la jeune fille garçonnière, un comportement scandaleux, qui ne saurait être celui d'une jeune bourgeoise», (cf. Lagevenais : « Le petit roman », Revue des Deux-Mondes, 1865, p.1861-1869).

${ }^{3}$ La novela Renée Mauperin se publicó del 3 de diciembre de 1863 al 10 de febrero de 1864 en el periódico l'Opinion Nationale con el título Mademoiselle Mauperin; el 27 de febrero aparece en la editorial Dubuisson una edición según la tipografía original, y ese mismo año, 1864, aparece ya como volumen en Charpentier con el título definitivo.

${ }^{4}$ Fechado 24 de enero 1875.
} 
también el objetivo buscado y deseado: pintar la joven moderna, tal y como es, por la influencia de la educación que ha ido recibiendo en los últimos 30 años.

Rénée Mauperin, est-ce le vrai, est-ce le bon titre de ce livre? La Jeune Bourgeoisie, le titre sous lequel mon frère et moi annoncions le roman avant qu’il fût terminé, ne définissait-il pas mieux l'analyse psychologique que nous tentions en 1864 de la jeunesse contemporaine? Mais à l'heure qu'il est, il est vraiment bien tard pour débaptiser le volume. Et, il m'est donné seulement aujourd'hui, de prévenir le lecteur que la fabulation d'un roman à l'instar de tous les romans, n'est que secondaire dans cette œuvre.

Les auteurs, en effet, ont, préférablement à tout, cherché à peindre, avec le moins d’imagination possible, la jeune fille moderne telle que l'éducation artistique et garçonnière des trente dernières années l'ont faite. Les auteurs se sont préoccupés, avant tout, de montrer le jeune homme moderne tel que le font au sortir du collège, depuis l'avènement du roi Louis-Philippe, la fortune des doctrinaires, le règne du parlementarisme (Goncourt, 1990 : 281).

Lo que pone de manifiesto el prefacio de Edmond son unas ideas y conceptos muy reveladores de las preocupaciones literarias, políticas y sociales de los Goncourt, de las que destacamos: el análisis psicológico, la educación artística de la época y la chica joven moderna; otro término que conviene subrayar es garçonnière, que sin duda merece ser considerado pues El Larousse de 1907 define este término así: «Qui appartient, qui convient aux garçons. Qui a des goûts, ou des habitudes de garçon, en parlant d'une fille». Evidentemente los Goncourt no señalan los aspectos que consideran como femeninos o masculinos pero el lector, y sobre todo el de la época, sabe claramente diferenciar los comportamientos, actitudes y modales correspondientes a cada género. En este sentido los autores han trabajado minuciosamente cada elemento de su texto, de la novela y del personaje para perfilar, mostrar y revelar el análisis psicológico llevado a cabo para sus fines literarios.

\section{Construir un personaje femenino}

La definición de «féminité» o «féminéité» remite al carácter de la mujer, de lo que es femenino, y si buscamos «féminiser» se entiende como: «prendre le type féminin». Construir pues literariamente un personaje femenino con actitudes y comportamientos «à la garçonnière» no es, desde nuestra perspectiva, nada evidente: analizaremos cómo los Goncourt consiguieron construir ese personaje femenino con rasgos masculinos a través de un fino análisis psicológico cuidando cada detalle y cada gesto.

\subsection{Blanche Passy, modelo de inspiración}

Para el personaje de Renata Mauperin, Edmond y Jules de Goncourt, grandes defensores del trabajo literario basado en el documento humano, buscaron un modelo del que inspirarse, una mujer de su entorno más cercano que les permitiera indagar en los aspectos más íntimos de su personalidad para así poder hacer un retrato fidedigno de la feminidad burguesa de su tiempo. En un principio empezaron dibujando una extensa red de datos sobre costumbres y tipos de la burguesía, dibujando perfiles y pequeñas anécdotas tomados en vivo y directamente de sus vivencias. Se ocuparon en un primer momento del tipo masculino, el joven burgués arribista, y así sucesivamente con cada personaje y cada detalle de la futura novela. Recopilaron cientos de datos y relatos breves de hechos los que sólo una mínima parte aparece en la novela. Su prima Blanche Passy sorprendentemente les trajo mucho más de lo deseado, dado que se sintieron totalmente fascinados por ella, mujer con mucha naturalidad y con unos encantos que rompían los esquemas y estereotipos impuestos por la sociedad del momento. En su conocido diario íntimo, Le Journal, Mémoires de la vie littéraire, Edmond y Jules describen a Blanche Passy como un hombre, un hombre honesto y lleno de cualidades que se mezclan de toques femeninos:

Blanche Passy, un homme, un honnête homme, avec la loyauté, la cordialité d’un homme ; des grâces de jeune fille dans cela ; un charme et un ami ; la raison mûrie et le cœur frais ; un esprit enlevé, on ne sait comment, du milieu bourgeois dans lequel il a été élevé ; toutes sortes d'aspirations au beau, au grand, au dévouement ; le mépris de ce 
qui est la pensée et l'entretien de la femme : le chiffon, l'argent, -a refusé quatre millions, -et la beauté de l'homme mésestimés (Goncourt, 1989 : 202).

Stéphanie Champeau, en su estudio sobre «Les Goncourt moralistes dans Renée Mauperin» señala que, aunque nunca lo afirmaran, seguramente también Edmond y Jules se inspiraron de las hijas de Théophile Gautier para el personaje de Renée ya que en las descripciones que hacen de ellas en Le Journal, se pueden detectar elementos comunes y coincidentes, y no debe extrañarnos pues a menudo sienten ante personas diferentes unas mismas emociones y describen actitudes y comportamientos similares ${ }^{5}$. Es muy interesante ver los elementos que adjudican al ser hombre y al ser mujer : los encantos, la razón, la aspiración a lo bello, la forma de pensar, los entretenimientos, la franqueza, son cualidades que en una mujer, la hacen más o menos femenina (entendiendo en su rol de mujer burguesa de finales del XIX) o será más masculina (alejándose del modelo de mujer); para construir sus personajes los Goncourt se basan en modelos vivientes, que han codeado y que han podido observar, y eso les sirve para asignarles determinadas características básicas en su primera redacción en vivo, que suelen hacer en su diario íntimo y después en sus novelas retoman esas impresiones que apuntaron para redactar y perfilar sus textos, técnica que exponen en el prefacio de Rénée Mauperin aunque de una forma general y abstracta: «les auteurs, en effet, ont, préférablement à tout, cherché à peindre, avec le moins d'imagination posible, la jeune fille moderne telle que l'éducation artistique et garçonnière des trente dernières années l'ont faite» (1990: 281). La anécdota, el documento humano, la observación son el esbozo de su trabajo literario artístico posterior. En lo que respecta al personaje de Renée y esos modelos vivientes que les inspiran, el lado «masculino» de esas mujeres es lo que destacan y asimismo sus sonrisas, miradas y cierto encanto que les cuesta definir.

\subsection{Renée, un personaje real}

Los Goncourt quieren construir con Renée un personaje femenino fuerte y consistente, capaz de llevar a cabo el análisis psicológico que, tal y como exponen en el prólogo, buscan forjar en la novela pues la parte de imaginación y de invención quieren dejarla de lado. A lo largo del texto y en diferentes escenas la joven Renée debe ir mostrando su verdadera forma de ser, desvelar su personalidad y a la vez ir barajando las expectativas de su familia y de su entorno, la clase burguesa de los años 1850. De esta forma un largo y minucioso retrato de la joven se perfila página a página. La joven Renée transgrede las prohibiciones impuestas por las costumbres y manifiesta excentricidades preocupantes: está dividida entre la voluntad de ser ella misma, sintiéndose libre, y lo que esperan de ella, lo conveniente para una chica joven de su clase social. En una carta a Flaubert los Goncourt confiesan lo que les está costando finalizar la novela por la dificultad y complejidad del personaje femenino que han puesto en primer plano:

Nous, mon cher Flaubert, nous travaillons sans travailler. Nous musons. Nous sommes dérangés par ce qui dérange et surtout par ce qui ne dérange pas. Et puis nous avons abordé une jeune fille bourgeoise de face, en plein : on glisse à tout moment dans les œufs à la neige ; et puis, peindre la bourgeoisie, c'est faire le tour d'une pièce de cent sous, on piétine sur place. Peut-être, parce qu'il est plat, notre roman s'allonge. Je vois encore au moins deux mois de noircissement de papier devant notre idée. Il y a à faire danser tout notre monde (Dufief, $1998: 106)$.

Renée Mauperin acaba siendo una heroína digna de admiración, porque su personaje busca salir de su medio burgués, sin saber muy bien cómo, aunque finalmente termine de manera bastante trágica. Dotada de un espíritu personal, lo cual ya no tienen ni los hombres según confiesan los Goncourt, huye de todo lo que le parece aburrido, inconsistente, sin vida y sin color. Renée va a ir apareciendo a lo largo del texto como un personaje que huye de la sociedad conformista, lo primero es apartarse de la sociedad de las mujeres que le parecen aburridas, se acerca a la de los hombres que le parece mucho más divertida y libre, y desde luego menos atada a lo conveniente. Renée rompe totalmente el esquema de la joven burguesa, encarnado en la novela por su hermana Henriette; hay en Renée una sensibilidad y una emoción

${ }^{5}$ Remitimos al Journal, 1989, p.791. 
muy particular propios de ella misma que le hacen desmarcarse de los demás personajes que la rodean. En este sentido es importante señalar que los Goncourt han construido un personaje femenino totalmente opuesto al personaje más revolucionario de la literatura francesa de finales del siglo XIX, el de la romántica Emma, esa mujer creada por Gustave Flaubert que fue un hito en las letras francesas, pero no cabe duda que Renée, ella, en su esencia más básica y elemental, es la perfecta anti-Bovary. Stéphanie Champeau comparte este mismo punto de vista y va más allá afirmando que Emma Bovary es totalmente antagónica a Renée (2008: 107). Los Goncourt construyen pues personajes basándose en seres reales, que han existido, de los que pueden retomar rasgos, aspectos, multitud de elementos que les da vida y así conforman el mundo de sus textos. Todo se construye, en cualquier caso, en función de una voluntad literaria y artística aunque -no debemos olvidarlo- hay un trasfondo social y político: «les auteurs se sont précoccupés, avant tout, de montrer le jeune homme moderne tel que le font au sortir du collège, depuis l'avènement du roi Louis-Philippe, la fortune des doctrinaires, le règne du parlementarisme» (Goncourt, 1990 : 281).

\section{Un diálogo inicial sorprendente}

Desde nuestro punto de vista la novela Renée Mauperin es un texto interesante y cautivador si lo leemos buscando detectar los elementos que los autores han buscado caracterizar como de «feminidad» en él. En este sentido el personaje de la joven Renée es quien adopta y traduce todos los aspectos y facetas que los autores quieren poner de manifiesto sobre la cuestión de la feminidad. El inicio de la novela, la primera escena del primer capítulo es indudablemente el episodio más provocador para nosotros por su presentación directa y abierta de varios elementos y por lo sorprendente de la aparición de otros muchos: nuestro trabajo ha consistido en analizar detenidamente su construcción y su temática para así poder determinar cuáles son los aspectos de la feminidad de Renée que los Goncourt consideraron como fundamentales y presentaron en primer plano. Así mismo hemos buscado entender cómo funciona la escritura Goncourt, cómo se construye y se forja, para obtener ese impacto, esa impresión a la vez natural y artista, abordando temas tan delicados como es la educación femenina -y por consiguiente la masculina- de la burguesía de finales del siglo XIX. Como la gran mayoría de las novelas de los Goncourt, Renée Mauperin comienza en medio de un diálogo, y todo el interés de concentra en el lenguaje de los personajes de quienes aún no conocemos las identidades y que no serán desveladas hasta que el lector esté muy adentrado en la discusión. El diálogo lo mantienen curiosamente un hombre y una mujer: la joven Renée y Reverchon, un amigo de Henri Mauperin, hermano de la protagonista. Ambos hablan de la vida, de la gente, de las contemporáneas de Renée y sin más entran en una charla íntima en la que Renée afirma abiertamente y sin sentirse coaccionada, que la compañía de sus contemporáneas le resulta bastante aburrida, pues los temas de conversación son muy limitados ya que sólo hablan del último sermón al que han asistido, del último concierto de piano que han estudiado o del último vestido que han llevado puesto, nada más. Con esta declaración de Renée sobre sus iguales, el lector se hace ya la idea de que Renée es una mujer que se desmarca de las demás, que se afirma a sí misma como una mujer con opinión propia y aficiones diferentes de las demás jóvenes, y que no acepta lo que le rodea sin más. Así es cómo Renée marca una primera distancia entre sus iguales a nivel social y de género: las conversaciones con las otras chicas le resultan aburridas, los temas abordados repetitivos y sus ocupaciones nada interesantes. Reverchon y Renée siguen con la conversación hablando de teatro, del éxito de la obra en boga en La Ópera cómica, y la charla nos muestra un claro panorama sobre las obras y teatros que le están permitidos y prohibidos a la joven Renée, y por consiguiente a las demás chicas, que por su juventud y su condición de mujer -aún soltera- no les están autorizados: ella se queja de lo injusto que es ser mujer, soltera o casada, joven o mayor, ya que nunca son libres de ir a los espectáculos, bailes y acontecimientos que desean. Renée, llevada por la emoción de tener un joven interlocutor que la escucha atentamente y a quien le puede contar cómo se siente, va a continuar con las quejas: bailar es también una actividad muy controlada y medida, no se puede elegir acompañante libremente ni se puede entablar ningún diálogo serio, sólo «pincer le monosyllabe» o sea decir sí o no; todo ello porque hay que tener un comportamiento «convenable». No puede sino sorprender al lector este íncipit de la novela: un hombre y una mujer hablan, ella critica la sociedad que la rodea (incluida su familia) él responde a sus preguntas y escucha; sin duda la amistad y la juventud compartidas les permite a ambos ciertas licencias, sin embargo el estatus social de ambos y la diferencia de sexo existente dejan clara muestra de que Renée es una joven osada y muy atrevida. Reverchon en cambio sólo se dedica a escuchar y a preguntar y no pone en tela de juicio nada... la actitud que adopta el acompañante es la de un oyente pasivo. 


\title{
3. Lo conveniente
}

Llegada Renée a este punto de afirmación sobre la falta de libertad de las mujeres en las actividades sociales (ir al teatro, hablar libremente, bailar, conversar) ahora se ocupa, en un segundo momento, de enumerar lo que resulta conveniente o totalmente inconveniente para una joven burguesa como ella ${ }^{6}$. El texto en sí resulta bastante repetitivo, dejando entrever el tono en el que debe estar hablando: queda pues prohibido hablar con hombres, es conveniente hablar con chicas de su sexo, leer es totalmente inconveniente, a cierta edad algunos folletines están permitidos pero nada de leer sobre crímenes u otros hechos; muy controladas también las artes, el piano, la pintura: nada de tocar piezas musicales a cuatro manos o más allá de la media, ni de dibujar con mina de plomo o aceite: la acuarela es la técnica aceptable para una joven burguesa. Lo conveniente es sinónimo de lo que conviene y obviamente lo que conviene a un grupo social o familiar no es forzosamente lo que construye un individuo en particular. A finales del siglo XIX las normas sociales están fuertemente instauradas en el bagaje cultural de la sociedad de la época y salirse de las normas es algo totalmente inaceptable y reprochable, el precio que se paga es el no ser integrado en los medios y círculos correspondientes y obviamente el individuo necesita de la sociedad; de ahí la fuerza y el atrevimiento de Renée que intenta reivindicar un nuevo espacio, un nuevo orden para las jóvenes mujeres. El nuevo orden que pide Renée es el de la libertad, libertad de hablar, libertad de crear, libertad de realizarse en tanto que mujer. La novela entera es una serie de episodios, diálogos y escenas llenas de decisiones y luchas que dibujan el verdadero personaje de Renée, mostrando situaciones que traducen la postura rebelde de Renée en la sociedad y en la familia Mauperin.

\section{En el elemento líquido}

Curiosamente, en medio de esta enumeración en tono de queja, Renée Mauperin exclama súbitamente a su interlocutor: «Mais il y a du courant ici, n’est-ce pas? On a peine à se tenir...», y es en ese preciso momento cuando el lector toma conciencia de que esta charla, entre la joven mujer y el joven burgués, sobre salidas, actividades, ocupaciones y restricciones de la vida de las señoritas, esta escena sobre lo aceptable y lo conveniente, y lo inaceptable e intolerable, tiene lugar en el agua. $\mathrm{Y}$ entonces sigue el texto con la frase del narrador que determina el decorado del relato: «Ceci était dit dans un bras de la Seine, entre la Briche et l'île Saint-Denis». A continuación todo un párrafo está dedicado a la descripción de ambos personajes charlando en el agua. Podemos entrever al final de este párrafo puramente descriptivo, un giro poético; de repente el texto se modifica y ya no es tan puramente descriptivo: Renée se transforma en una escultura, en una diosa del mar, viva, pues el movimiento del río y su frialdad le transmiten «algo de la ondulación del agua». He aquí una muestra del estilo propio de los Goncourt, un tono moderado de la escritura artista pues aún no está en todo su esplendor. De repente, con toda inocencia, la joven Renée constata que se encuentra con un hombre, en el agua del Sena, los dos cuerpos frente a frente, y entonces siente cierto pudor... y retoma la palabra:

\begin{abstract}
Ah ! voilà, par exemple, reprit-elle, ce qui ne doit pas être convenable du tout, de nager avec vous... Nous serions aux bains de mer, ce serait bien différent. Nous aurions des costumes absolument comme ça... Nous descendrions d'une cabine comme nous sommes descendus de la maison. Nous aurions marché sur la plage comme nous avons marché sur la berge... Nous serions dans l'eau jusque-là, absolument comme ici... La vague nous roulerait de la même façon que ce courant... Mais ce ne serait plus du tout la même chose, plus du tout : l'eau de la Seine n'est pas convenable ! (Goncourt, 1990 : 53-54)
\end{abstract}

Bañarse con Reverchon en el río Sena es totalmente inconveniente, en el mar, sin embargo, sería muy diferente. Bañarse en el mar, pasearse hablando por la playa sería una actividad en sociedad, un lugar frecuentado por más gente, y no un cuerpo a cuerpo entre dos personas, de sexo opuesto, en un lugar alejado. Y no hay que olvidar la vestimenta, que tampoco es la adecuada. De repente Renée cambia de conversación, como si abordando otros temas (la hora de la comida, el círculo de amigos, ...) pudiera hacer de la situación algo más aceptable. Esta vez es la propia Renée quien se ha percatado que no está actuando según su estatus y su condición. El diálogo de nuevo se interrumpe, esta vez por la

\footnotetext{
${ }^{6}$ El Larousse pour tous de 1907 define el término «Convenable» : adj. Sortable, qui convient : mariage convenable. Qui est à propos, expédient : récompense convenable. Décent, bienséant : cela n'est pas convenable. Ant. Inconvenant, malséant, déplacé. Si buscamos «Déplacé, e» : adj. Qui n'est pas à la place qui lui convient : être déplacé dans une société. Fig. Qui manque aux convenances : propos déplacés.
} 
llegada de Henri y de sus amigos con una barca, y Renée intenta atraer la mirada de Reverchon hacia el paisaje que les rodea. Primero describe con un lenguaje puramente pictórico el cielo y después las orillas. Renée se siente llevada por la belleza de todo cuanto les rodea, belleza no compartida por Reverchon, hay que decirlo. Aquí la descripción sigue en el estilo Goncourt, un estilo mucho más artista e impresionista: Edmond y Jules no han podido resistirse, la descripción está construida a través de la mirada de Renée, ella sabe apreciar la luz, los contrastes, los colores, los olores, los ruidos... ella habla de su sentir personal respecto al arte y al paisaje que les rodea. Pero no hay ningún indicio de atracción ni de sentimientos entre ellos, al contrario, los movimientos de Renée en el agua sólo pueden ser interpretados por el lector como unos movimientos de mujer, en el elemento líquido, con gestos sensuales que dejan entrever algo más que lo puramente inconveniente: son una transgresión, y sin embargo, ninguna muestra, ninguna insinuación sobre la inocente postura de Renée.

\subsection{Lo femenino frente a lo masculino}

En su discurso Renée pone en evidencia ante todo las diferencias tan abismales que existen entre hombres y mujeres. Evidentemente el hombre es mucho más libre en sus actividades, en sus movimientos, en sus palabras. Ser mujer a mediados del siglo XIX conlleva muchas ataduras por las expectativas sociales. En algunas de sus afirmaciones percibimos cierto celo de Renée por la condición masculina de Reverchon, sin embargo no hay duda de que Renée se siente bien siendo mujer: en la descripción que hace del paisaje, en su manera de juzgar su entorno, ella se siente feliz por ser luchadora y por conseguir que ella sea así y su familia y amigos la acepten como es. Ella es feliz pintando con la mirada, -al igual que los hermanos Goncourt- sus palabras también le ayudan a decorar y a crear impresiones. Qué duda cabe que Renée exterioriza a través de sus gestos y sus palabras que se siente libre, en el agua, libre de hablar, de pintar con la mirada y las palabras, el agua la libera de las imposiciones sociales y familiares. No debemos olvidar enlazar Renée con sus modelos reales, puesto que ese encanto de creación poética sin duda viene inspirado por Blanche Passy: recordemos que siempre los Goncourt se atan a un referente objetivo para sus personajes. Vemos retratada a una Renée desde el exterior pero sin duda podemos adivinar su mundo interior, a través de sus palabras y gestos. Dibujan el retrato de una mujer que busca escapar del mundo burgués en el que está inmersa: gracias a esta escena del baño Renée se muestra espontánea, viva, entusiasta, franca y honesta con ella misma. El agua es pues el lugar de la liberación lingüística, emocional y sensorial de Renée. El agua del Sena aunque no sea conveniente, es liberadora para la mujer que está encerrada en ese personaje que encarna Renée Mauperin, y ello no es socialmente conveniente. Todo elemento liberador en diferentes planos es lo que le opone a los jóvenes de la época. La mujer no debe sentir, vivir las palabras, las emociones ni las sensaciones, solamente está para adornar y cumplir con lo establecido.

\section{Un estilo artista y la marca Goncourt}

Sin duda el aspecto más polémico para la crítica fue esta escena del baño; el lenguaje utilizado por el personaje de Renée, sus diferentes posturas, movimientos, su actitud, todas las libertades que se permite, tanto en sus afirmaciones como en sus gestos, son una provocación. ¿Cómo podemos interpretar esta escena dentro de la producción goncourtiana?, ¿qué sentido adopta este personaje con todo y cada uno de los elementos que le han otorgado? ¿Se trata de una provocación, de una osadía puestas así de cualquier forma en el inicio del libro para simplemente llamar la atención del lector? Edmond y Jules, como buenos observadores de la realidad, como buenos periodistas saben perfectamente que el personaje de Renée no es ni se corresponde a ninguna joven de la burguesía, a ninguna mujer de su época incluso: jamás se comportaría como la retratan, ni en sus gestos, ni en sus actitudes y menos aún con el lenguaje que adopta. Entonces los Goncourt construyen un personaje femenino totalmente suyo: se toman el derecho de crear, en nombre a la verdad, una mujer con su propia forma de ser, con su lenguaje y la libertad de moverse y ser como ella lo siente y en cualquiera de sus actos se traduce siempre algo de provocación. Salirse de lo establecido, regulado y normativo siempre requiere valentía y es interpretado como provocador. Hay pues que abanderar en el personaje de Renée esa faceta de los Goncourt a quienes les gusta llamar la atención: Renée es la hija creada y creativa de Edmond y Jules: en ella están todas las cualidades que según ellos deberían tener las mujeres: sentirse libres en una faceta artista que les permita disfrutar de los paisajes y colores que le rodean; sentir la profundidad del agua, la luz que flota, los reflejos, ... todo lo que a ellos les hace felices y sentir vivos quieren que el personaje de Renée también sepa sentirlo y transmitirlo. Es el primer personaje que crean con tanta vida, que se maravilla ante la belleza e insiste en querer 
transmitirlo: «Si, c'est beau! Je vous assure que c'est beau...». Sin duda Blanche Passy le ha forjado al personaje de Renée todos sus encantos, pero Edmond y Jules le inculcan a su heroína también todo aquello que les había fascinado de Blanche, y ello a lo largo de toda la novela: los decorados, los objetos... cada elemento surge de algo descubierto y vivido en la vida de Blanche. Es pues esta primera escena de la novela, la más excepcional, la del baño en el Sena, que provocó el mayor escándalo tanto por su audacia como por su supuesta inconveniencia en la postura de Renée, que con tanta familiaridad y frescura Renée toma y repite con placer, franqueza y alegría.

La combinación de los elementos lingüísticos de l'écriture artiste de los Goncourt subraya la importancia de la presencia del agua y del mundo imaginario que evoca, que son por si mismos también elementos transgresores y evocadores de lo prohibido para las chicas jóvenes de la época. El agua es el lugar donde surgen y se liberan las emociones, el lector asiste pues a una escena de un resurgir de una mujer diferente como si de un nuevo nacimiento se tratara, lo nuevo que surge es: la liberación emocional de Renée.

\section{Referencias bibliográficas}

BARon, Philippe (1997). «Renée Mauperin, pièce d’Henry Céard, tirée du roman des Goncourt». Cahiers Edmond \& Jules de Goncourt, n 5, p. 82-99.

Champeau, Stéphanie (2008). «Les Goncourt moralistes dans Renée Mauperin». Cahiers Edmond \& Jules de Goncourt, n 15, p. 95121.

DuFIEF, Pierre-Jean (1998). Flaubert Goncourt Correspondance. Paris: Flammarion.

Goncourt, Edmond et Jules (1989). Journal. Mémoires de la vie littéraire 1851-1865. Paris: Robert Laffont.

GonCOURT, Edmond et Jules (1990). Renée Mauperin. Paris: Flammarion.

RicATtE, Robert (1953). La création romanesque chez les Goncourt, 1851-1870. Paris: Armand Colin. 